\title{
Three-Dimensional Magnetization Reversal Measurements in Nanoparticles
}

\author{
E. Bonet,,${ }^{1} *$ W. Wernsdorfer, ${ }^{1}$ B. Barbara,${ }^{1}$ A. Benoit, ${ }^{2}$ D. Mailly, ${ }^{3}$ and A. Thiaville ${ }^{4}$ \\ ${ }^{1}$ Laboratoire Louis Néel-CNRS, BP166, 38042 Grenoble, France \\ ${ }^{2}$ CRTBT-CNRS, BP166, 38042 Grenoble, France \\ ${ }^{3}$ L2M-CNRS, 196 avenue H. Ravera, 92220 Bagneux, France \\ ${ }^{4}$ LPS-Université Paris-Sud, 91405 Orsay, France
}

(Received 1 July 1999)

\begin{abstract}
The first three-dimensional switching field measurements of an individual nanometer-sized magnetic particle are presented. The angular dependence of the switching field can be described by the model of uniform rotation of magnetization. However, the anisotropy function proposed by Stoner and Wohlfarth appears to be simplistic. In order to reproduce the measured switching fields, one has to take into account higher order anisotropy terms. These terms are very important to understand dynamical magnetization reversal properties in the classical and the quantum regimes.
\end{abstract}

PACS numbers: 75.50.Tt, 75.30.Gw, 75.60.-d

Since the late 1940s, nanometer-sized magnetic particles have provoked continuous interest because studying their properties has proved to be scientifically and technologically very challenging. The magnetization reversal of such particles had been described more than 50 years ago by Stoner and Wohlfarth [1] and Néel [2] in a very simple theory: the uniform rotation model. Despite intense activities during the past few decades, the difficulties in preparing nanoparticles of good enough quality slowed down the progress in this field. In the past few years, this has no longer been the case because of the emergence of new fabrication techniques which have allowed the fabrication of small objects with the required structural and chemical qualities. In order to study these mesoscopic objects, new techniques were developed such as magnetic force microscopy [3], magnetometry based on micro-Hall probes [4,5], micro-SQUIDs [6], or magnetometry based on spindependent tunneling with Coulomb blockade [7]. These techniques lead to a new understanding of the magnetic behavior of nanoparticles, which is very important for the development of new fundamental theories of magnetism and for modeling new magnetic materials for permanent magnets or high density recording.

One of the important fundamental questions is whether the model of Stoner and Wohlfarth, widely used in magnetism, provides a realistic description of the magnetization reversal in a magnetic nanoparticle. In order to demonstrate experimentally the uniform rotation mode, the angular dependence of the magnetization reversal has often been studied. First, it is important to choose a proper system which does not present nonuniform magnetization states. Then one has to carry out single particle measurements in order to get rid of distributions of anisotropies. Finally, the angular dependence of the switching field has to be measured for all directions in the field space. In the Stoner-Wohlfarth model, the graph of the angular dependence in polar coordinates gives the well-known "StonerWohlfarth astroid." In this Letter, we show that new techniques of single particle measurements [3,5-7] and, in particular, the micro-SQUID technique [6] allow one to test accurately the model of uniform rotation, not only in a given plane but also in all directions of the field space. These measurements give a geometrical representation of the magnetic anisotropy of a given nanoparticle. We demonstrate that the 2D Stoner-Wohlfarth model, which is continuously used in both basic and applied research in magnetism, constitutes an oversimplified approach which should be overcome if one wants to understand deeply the physical mechanisms of magnetization reversal. We show that the generalization of the Stoner-Wohlfarth model, recently proposed by Thiaville [8], is well suited to interpret our experimental results.

The uniform rotation model is the simplest model describing the magnetization reversal of nanoparticles. It assumes that the nanoparticle is small enough so that exchange interactions lead to uniform magnetization within the particle. It can be written $M_{s} \mathbf{r}$, where $M_{s}$ is the saturation magnetization and $\mathbf{r}$ is a unit vector.

The only magnetic degrees of freedom of the particle are those of the orientation of $\mathbf{r}$. The dynamics of the magnetization is described using a potential energy $V(\mathbf{r}, \mathbf{H})$ depending on the orientation of $\mathbf{r}$ and on the applied field $\mathbf{H}$. At zero applied field, this energy $V_{0}(\mathbf{r})$ is called anisotropy energy. It has at least three sources: shape, surface, and magnetocrystalline anisotropy.

Shape anisotropy comes from the anisotropy of the demagnetizing field. It is in fact the remaining effect of dipolar interaction when the magnetization vector $M_{S} \mathbf{r}$ is the only degree of freedom. Like dipolar interaction, shape anisotropy is a quadratic form of the magnetization. It is therefore highly symmetrical with respect to $\mathbf{r}$, having three symmetry planes orthogonal to each other.

Surface anisotropy is expected to be essentially of second order and can therefore be treated as a contribution to the shape anisotropy.

Nanometer-sized samples are often single crystalline. Then, they have a well-defined magnetocrystalline anisotropy arising from the coupling of the magnetization with 
the crystal lattice. In contrast to shape anisotropy, magnetocrystalline anisotropy can be a function of $\mathbf{r}$ of any order. It needs only to have the symmetries of the crystal. Since these symmetries are in general different from those of the shape anisotropy, the total resulting anisotropy symmetry is very low: only central symmetry is granted.

An external magnetic field $\mathbf{H}$ adds a Zeeman contribution to the potential energy. Hence, the total energy is

$$
V(\mathbf{r}, \mathbf{H})=V_{0}(\mathbf{r})-v \mu_{0} M_{s} \mathbf{r} \cdot \mathbf{H},
$$

where $v$ is the volume of the particle.

At zero kelvin, $\mathbf{r}$ is in a local minimum of $V$. If the applied field $\mathbf{H}$ is continuously changed, $V$ and the position of the local minimum vary continuously as well. The orientation $\mathbf{r}$ of magnetization follows the position of the minimum. However, there are particular fields where the minimum will disappear by merging with a saddle point. When this happens, $\mathbf{r}$ varies discontinuously and jumps to another minimum of $V$. This jump, called magnetization reversal, is illustrated in Fig. 1. The corresponding fields are called switching fields, and the locus in the field space of all the switching fields is a 2D manifold called critical surface. Knowing $V_{0}$, the critical surface can be computed easily by searching the fields at which a minimum of $V$ disappears [8].

The original Stoner-Wohlfarth model [1] is a special case of the model above obtained by assuming the simplest nontrivial form for magnetic anisotropy:

$$
V_{0}=-\frac{K}{2} v \cos ^{2} \Theta,
$$

where $K$ is a positive constant called magnetic anisotropy constant, $v$ is the particle volume, and $\Theta$ is the angle between $\mathbf{r}$ and the easy axis of magnetization. By assuming this axial anisotropy symmetry, the problem of magnetization reversal becomes essentially a $2 \mathrm{D}$ problem. The corresponding critical surface is the well-known StonerWohlfarth astroid when computed in two dimensions. To get the whole 3D surface, one just has to rotate the astroid around its easy axis, restoring the third dimension of the problem.
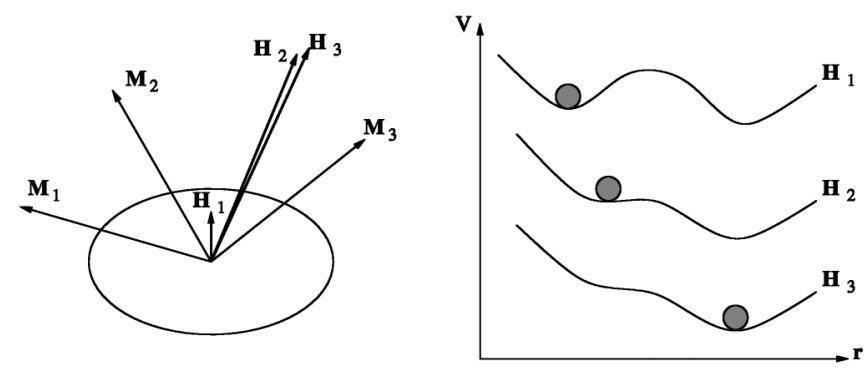

FIG. 1. To the left, scheme of magnetization directions for different fields. The magnetization varies continuously as the field goes from $\mathbf{H}_{1}$ to $\mathbf{H}_{2}$, but it varies abruptly when $\mathbf{H}$ goes from $\mathbf{H}_{2}$ to $\mathbf{H}_{3}$. To the right, the potential $V(\mathbf{r})$ is shown against an arbitrary coordinate of $\mathbf{r}$ for these three particular fields.
The interesting point about this model is its simplicity. It describes a magnetic particle using only two parameters: the orientation of the easy axis with respect to the field and the strength $K$ of the anisotropy. The magnetization lies in the plane defined by the anisotropy direction and the field. Because of this simplicity, it has been widely used for studying large assemblies of nanoparticles where one needs to have few parameters in order to be able to get their distribution by fitting experimental data. Since 3D astroid measurements were not possible until now, there was no need for more sophisticated models. Since this is possible now, one has to go further than the usual 2D StonerWohlfarth model, especially because in real particles the anisotropy is more complex and less symmetrical than in this model, even if only the shape anisotropy is taken into account.

By using the micro-SQUID technique [6], we measured magnetization switching fields of barium ferrite particles: $\mathrm{BaFe}_{12-2 x} \mathrm{Co}_{x} \mathrm{Ti}_{x} \mathrm{O}_{19} \quad(x=0.8)$, which we will call $\mathrm{BaFeO}$, in the size range of $10-20 \mathrm{~nm}[9,10]$. In comparison to previous measurements $[11,12]$, we improved the micro-SQUID technique. Since a SQUID works exclusively when the applied field is in the plane of its loop, we were limited to measure only a cross section of the whole critical surface. We overcame this problem by using a twostep method. In the first step, the SQUID is switched off and a field is applied in an arbitrary direction which may or may not cause a magnetization switching. Then, the SQUID is switched on and a second field is applied in the plane of the SQUID to probe the resulting magnetization state. This method allows us to scan the entire field space.

Figure 2 shows the entire critical surface measured at $50 \mathrm{mK}$ on a $\mathrm{BaFeO}$ particle. This particle is about $20 \mathrm{~nm}$ wide and has hexagonal symmetry. Although it has a simple critical surface being similar to the original StonerWohlfarth "astroid," it cannot be generated by the rotation of a 2D astroid. However, taking into account shape anisotropy and hexagonal crystalline anisotropy of $\mathrm{BaFeO}$, we are able to find a good agreement with a 3D StonerWohlfarth model (see Fig. 3). The anisotropy function is

$$
\begin{aligned}
\frac{2 V_{0}(\mathbf{r})}{v M_{s}}= & -A r_{z}^{2}+B r_{y}^{2}+C\left(r_{x^{\prime}}^{2}+r_{y^{\prime}}^{2}\right)^{2} \\
& +D\left(r_{x^{\prime}}^{2}+r_{y^{\prime}}^{2}\right)^{3},
\end{aligned}
$$

where $\left(r_{x}, r_{y}, r_{z}\right)$ are the coordinates of $\mathbf{r}$ in the coordinate system that diagonalizes the quadratic part of $V(\mathbf{r})$, and $\left(r_{x^{\prime}}, r_{y^{\prime}}, r_{z^{\prime}}\right)$ are its coordinates in a coordinate system where the $z^{\prime}$ axis is the crystal sixfold axis. The values of the anisotropy constants are $A=347.2 \mathrm{mT}, B=3.5 \mathrm{mT}$, $C=29.5 \mathrm{mT}$, and $D=-7.0 \mathrm{mT}$.

Notice that the magnetocrystalline part of the anisotropy function exhibits rotational symmetry around $z^{\prime}$. It is possible to consider a less symmetrical term with only hexagonal symmetry, but this seems to be useless since the critical surface shows no evidence for such a symmetry. 

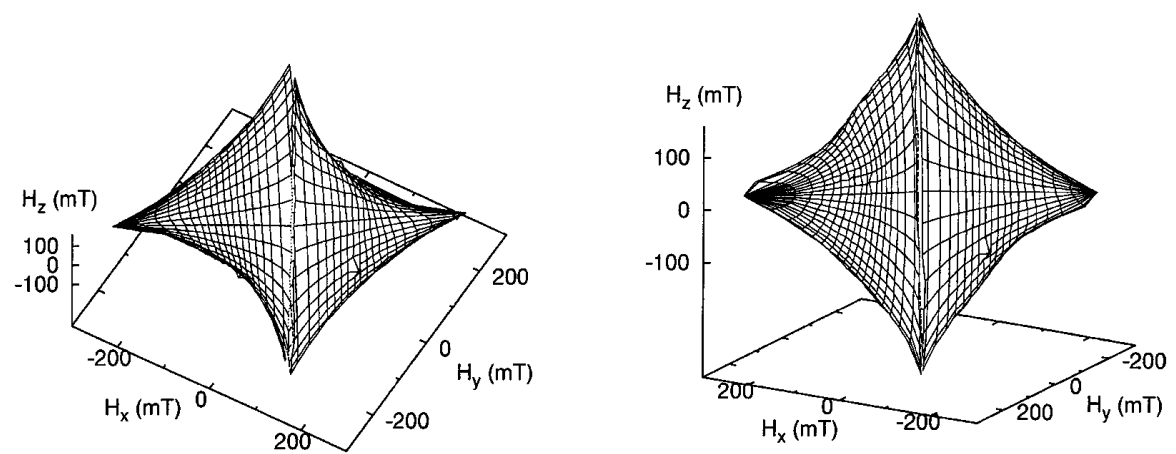

FIG. 2. Critical surface of a $\mathrm{BaFeO}$ nanoparticle as measured in three dimensions and seen from two orthogonal points of view. While about 4000 points were measured on the surface, only a few hundred are represented on this figure.

Furthermore, the axes $x, y$, and $z$ are not directly related to the crystal axes, nor to the shape anisotropy axes because both shape and crystalline anisotropies contribute to the quadratic part of the total anisotropy. Therefore the axes $x, y$, and $z$ are the directions of the eigenvectors of the sum of these two contributions.

As can be seen in Fig. 2 and from the values of the anisotropy constants, the effective anisotropy of this particle is mainly quadratic. However, higher order terms may play an important role in some circumstances, for example, in the splitting of quantum energy levels. This has to be taken into account when studying the possibility of a quantum tunneling of the magnetization.
We have shown experimental results which can be explained by the uniform rotation model for a classical system at zero temperature and in three dimensions. To our knowledge, this is the only model which gives a simple explanation of experimentally obtained 3D StonerWohlfarth astroids. This kind of study can be theoretically and experimentally extended in two directions. On the one hand, one can consider a system at nonzero temperature [11]. On the other hand, one may want to consider possible quantum effects at such "macroscopic" dimensions [12]. Both extensions lead to the same kind of results. Namely, the switching field becomes a stochastic variable and the magnetization switching takes place before the energy
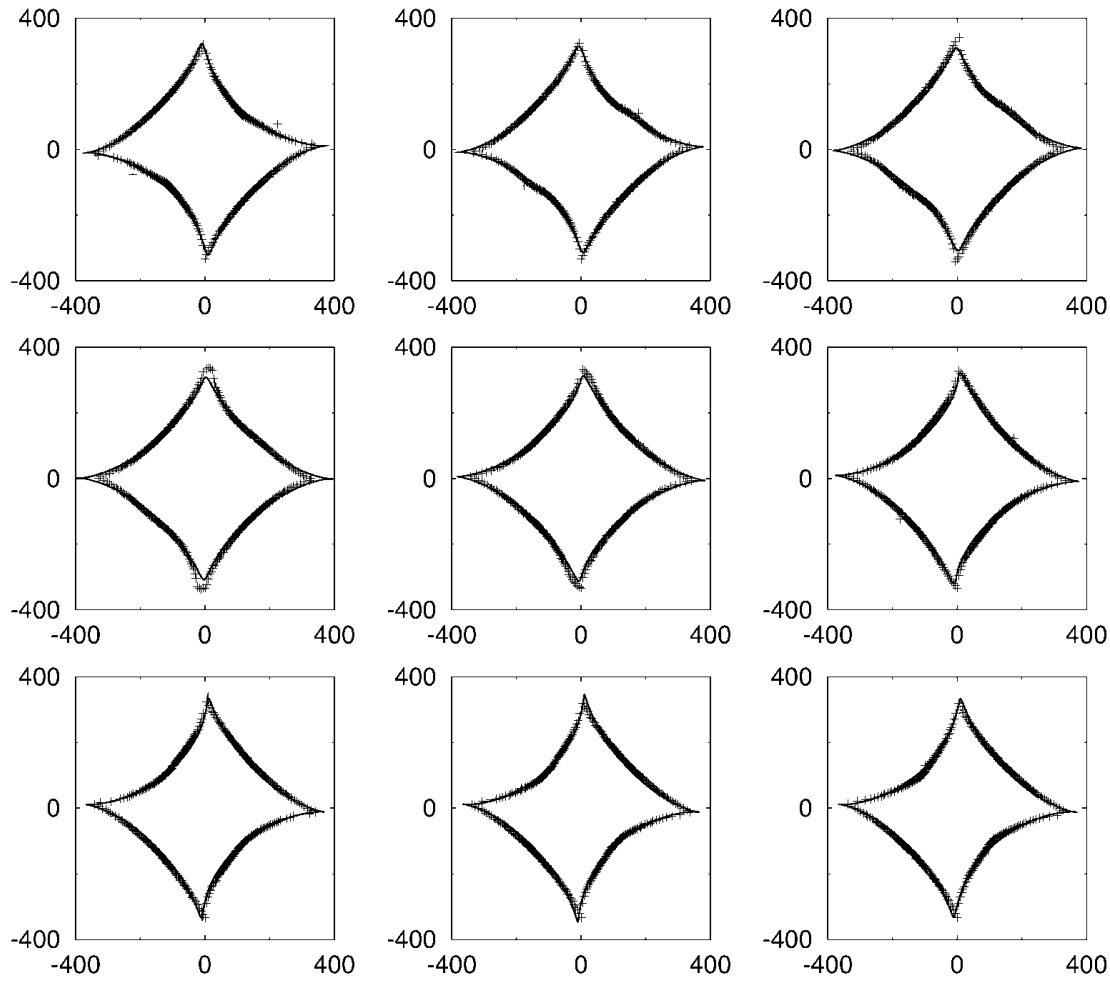

FIG. 3. Nine different cross sections of the critical surface showing the measured points (same data as from Fig. 2) and the fitted curve. All these cross sections share a common $y_{0}$ axis along the cusp of the astroid (shown vertically). Fields are given in mT. The section planes are spaced $20^{\circ}$ from one another. 
barrier goes to zero. This can be due to thermal activation or to quantum tunneling of the magnetization.

We have studied experimentally the dynamics of the magnetization reversal at finite temperatures. A detailed knowledge of the anisotropy function $V_{0}(\mathbf{r})$ is necessary to interpret these experiments. This may also be the case for particles assemblies. In general, $V_{0}(\mathbf{r})$ is supposed to be the simple 2D Stoner-Wohlfarth potential. However, this form of $V_{0}(\mathbf{r})$ is a very special and a highly symmetrical case. Some results obtained with this model depend on rotational symmetry, which does not exist in real systems. Being able to measure the effective anisotropy function of a given nanoparticle, we prove here that it is possible (and we think, preferable) to start with arbitrary anisotropy functions and determine them by fitting switching field measurements.

One of the most exiting points about the classical theory at nonzero temperature is that there is a relation between the characteristic time $\tau_{0}$ of the dynamics, the damping parameter in the dynamical equation of $\mathbf{r}$, and the anisotropy function $V_{0}(\mathbf{r})$ [13]. Knowing the effective damping parameter in small particles is very important for the research on ultrafast magnetic recording systems. Since $\tau_{0}$ can be obtained from dynamical measurements, the knowledge of the real anisotropy function opens the way to the experimental determination of the damping parameter of single nanoparticles, and also to test the different theories dealing with intrawell fluctuations in both classical and quantum regimes [14].
We acknowledge O. Kubo and H. Nakano, Toshiba Corporation, for sample preparation. This work has been supported by DRET and Rhône-Alpes.

*Email address: bonet@ polycnrs-gre.fr

[1] E. C. Stoner and E. P. Wohlfarth, Philos. Trans. R. Soc. London A 240, 599 (1948); reprinted in IEEE Trans. Magn. 27, 3475 (1991).

[2] L. Néel, Ann. Geophys. 5, 99 (1949).

[3] M. Lederman, S. Schultz, and M. Ozaki, Phys. Rev. Lett. 73, 1986 (1994).

[4] A.D. Kent, S. von Molnar, S. Gider, and D. D. Awschalom, J. Appl. Phys. 76, 6656 (1994).

[5] J. G. S. Lok et al., Phys. Rev. B 58, 12201 (1998).

[6] W. Wernsdorfer et al., Phys. Rev. Lett. 77, 1873 (1996).

[7] S. Guéron, M. M. Deshmukh, E. B. Myers, and D.C. Ralph, cond-mat/9904248.

[8] A. Thiaville (to be published).

[9] O. Kubo, T. Ido, H. Yokoyama, and Y. Koike, J. Appl. Phys. 57, 15 (1985).

[10] O. Kubo, T. Ido, and H. Yokoyama, IEEE Trans. Magn. 23, 3140 (1987).

[11] W. Wernsdorfer et al., Phys. Rev. Lett. 78, 1791 (1997).

[12] W. Wernsdorfer et al., Phys. Rev. Lett. 79, 4014 (1997).

[13] W. T. Coffey et al., Phys. Rev. Lett. 80, 5655 (1998).

[14] D. A. Garanin, cond-mat/9905010 (to be published). 